\title{
Reply: Lack of evidence supporting a role for DPP6 sequence variants in Alzheimer's disease in the European American population
}

\author{
Rita Cacace ${ }^{1,2}\left(\right.$ C) $\cdot$ Christine Van Broeckhoven ${ }^{1,2}$ (1)
}

Received: 22 January 2021 / Revised: 22 January 2021 / Accepted: 23 January 2021 / Published online: 16 February 2021

(c) The Author(s) 2021

We appreciate the work performed by Kirola et al. [3] in attempting to replicate our genetic findings in DPP6 [1]. Kirola et al. investigated association of DPP6 with Alzheimer's disease (AD) in whole exome sequencing (WES) data of three cohorts of non-Hispanic white patients and matched controls [3]. Two cohorts included late-onset AD patients: FASe, with 1212 patients and 341 controls and ADSP with 5656 patients and 4601 controls. The third cohort included 1385 early-onset (EO)AD patients and 3864 controls. The authors conclude that there is no association of DPP6 with $\mathrm{AD}$, not with single variants or in gene burden tests. [3]. The authors hypothesize a population specific effect of DPP6 in our study [3]. We read the work of Kirola et al. with great interest, but it seems to us that their interpretation of the data is not providing a comprehensive vision of the genetic role of $D P P 6$ in $\mathrm{AD}$.

We discovered DPP6 in an AD family linked to chromosome $7 \mathrm{q} 36[1,6]$. Using whole genome sequencing (WGS), we identified a chromosomal inversion disrupting the DPP6 coding sequence, leading to haploinsufficiency as disease mechanism [1]. Kirola et al. used WES data which is a limiting factor for observing structural variants [3]. We also screened DPP6 in AD patients with an onset age $\leq 70$ years and in patients with frontotemporal dementia (FTD).We identified an enrichment of rare non-synonymous variants (nonsense, frameshift and missense) in the EOAD cohort ( $p$ value $=0.03, \mathrm{OR}=2.2195 \%$ CI $1.05-4.82)$, and the FTD cohort $(p$ value $=0.006, \mathrm{OR}=2.59,95 \%$ CI $1.28-5.49)$ [1] We applied a SKAT-O rare variant gene-based test, accounting for pathogenic, non-pathogenic, and even protective variants in our statistical model as it is widely accepted that also causal genes harbor benign variants [2]. It is not

Christine Van Broeckhoven

christine.vanbroeckhoven@uantwerpen.vib.be

1 VIB Center for Molecular Neurology, Antwerp, Belgium

2 Department of Biomedical Sciences, University of Antwerp, Antwerp, Belgium unlikely that some of the variants identified by Kirola et al. are benign. We uncovered the benign effect of the p.Ala778Thr, which we [1] and Kirola et al. [3] found in patients and controls. Regrettably, the overlap of rare nonsynonymous variants between the two studies is limited, seven in the ADSP and none in the EOAD cohorts as we identified a p.Ala655Val [1] instead of the p.Ala655Thr that Kirola et al. reported [3]. Also, the lack of overlap of variants in exon 1, might be consistent with a lower quality of the WES reads in this region. We used Sanger sequencing to overcome this issue [1]. Further, it is not clear how the single variants were selected for inclusion or exclusion from the burden tests (Tables $\mathrm{S} 2$ and $\mathrm{S} 3$ versus Table $\mathrm{S} 1$ in the paper of Kirola et al. [3]). It seems to us that the inclusion criteria were not entirely followed.

Based on our results in the DPP6 family [1], we predict that loss of function $(\mathrm{LoF})$ variants (structural variants e.g., inversion; mutations leading to premature termination codons (PTCs)), present with a higher penetrance and disease impact, contributing to DPP6 haploinsufficiency. But LoF mutations are extremely rare compared to missense mutations, which might have a variable risk contribution to disease depending on DPP6 expression levels [1]. Kirola et al. identified in DPP6 a frameshift variant, p.His431fs, and a variant in a donor splice-site (hg19 g.154664404G > A), affecting all protein coding DPP6 isoforms [3]. The variants were found in two AD patients in the ADSP cohort and never in 8806 controls pooled from the three control cohorts. These findings of Kirola et al., support the predicted functional mode of action of DPP6 [1]. Notably, the p.His $431 \mathrm{fs}$ appears to be excluded from both burden tests (MAF $\leq 1 \%$ and CADD $\geq 20$ ) and is absent from Tables S2-S3 even though it has a $\mathrm{MAF} \leq 1 \%$ and we calculated a CADD score of 32 for it. Also, the donor splice-site variant is included only in the burden test of variants with a CADD score $\geq 20$ (Table S3) while the variant has a MAF $\leq 1 \%$ [3].

To complete, the genetic association of DPP6 with FTD is shown in an independent study which analyzed WGS data 
of individuals from 23 different sites from Europe, North America, and Australia [5], arguing against a population effect in Belgium. Also, in the latter study, a truncating mutation in DPP6 was observed in one patient, leading to partial loss of DPP6 expression in the brain [5]. We agree that replication of the rare variant association in independent cohorts is fundamental, but we stress that functional evidence is crucial in case of novel disease genes, to understand how they contribute to a specific phenotype. A recent work suggests a neuroprotective role for DPP6 [4]. In aged Dpp6 knockout mice the loss of protein is shown to lead to the enhanced formation of abnormal, enlarged presynaptic terminals [4]. These structures partly colocalize with proteins associated with $\mathrm{AD}$, including $\mathrm{A} \beta$, pTau and chromogranin A [4].

We conclude that the presented work by Kirola et al. shows a lack of replication of our study, but cannot conclude that there is no association of DPP6 with $\mathrm{AD}$ [3], since their study is limited to statistical analysis of rare coding variants without further interpretation of a potential functional effect and the type of variants.

Open Access This article is licensed under a Creative Commons Attribution 4.0 International License, which permits use, sharing, adaptation, distribution and reproduction in any medium or format, as long as you give appropriate credit to the original author(s) and the source, provide a link to the Creative Commons licence, and indicate if changes were made. The images or other third party material in this article are included in the article's Creative Commons licence, unless indicated otherwise in a credit line to the material. If material is not included in the article's Creative Commons licence and your intended use is not permitted by statutory regulation or exceeds the permitted use, you will need to obtain permission directly from the copyright holder. To view a copy of this licence, visit http://creativecommons.org/licenses/by/4.0/.

\section{References}

1. Cacace R, Heeman B, Van Mossevelde S, De Roeck A, Hoogmartens J, De Rijk P et al (2019) Loss of DPP6 in neurodegenerative dementia: a genetic player in the dysfunction of neuronal excitability. Acta Neuropathol 137:901-918. https://doi. org/10.1007/s00401-019-01976-3

2. Cruts M, Theuns J, Van Broeckhoven C (2012) Locus-specific mutation databases for neurodegenerative brain diseases. Hum Mutat 33:1340-1344. https://doi.org/10.1002/humu.22117

3. Kirola L, Budde JP, Wang F, Norton J, Morris JC, NIA-LOAD family study group et al. (2021) Lack of evidence supporting a role for DPP6 sequence variants in Alzheimer's disease in the European American population. Acta Neuropathol Accepted

4. Lin L, Petralia RS, Lake R, Wang YX, Hoffman DA (2020) A novel structure associated with aging is augmented in the DPP6KO mouse brain. Acta Neuropathol Commun 8:197. https://doi. org/10.1186/s40478-020-01065-7

5. Pottier C, Ren Y, Perkerson RB 3rd, Baker M, Jenkins GD, van Blitterswijk M et al (2019) Genome-wide analyses as part of the international FTLD-TDP whole-genome sequencing consortium reveals novel disease risk factors and increases support for immune dysfunction in FTLD. Acta Neuropathol. https://doi. org/10.1007/s00401-019-01962-9

6. Rademakers R, Cruts M, Sleegers K, Dermaut B, Theuns J, Aulchenko $Y$ et al (2005) Linkage and association studies identify a novel locus for Alzheimer disease at 7q36 in a Dutch population-based sample. Am J Hum Genet 77:643-652. https://doi. org/10.1086/491749 (S0002-9297(07)61012-X[pii])

Publisher's Note Springer Nature remains neutral with regard to jurisdictional claims in published maps and institutional affiliations. 